\title{
Padrão Sintomatológico em Pacientes do Câncer Colorretal de acordo com a Idade
}

\author{
doi: https://doi.org/10.32635/2176-9745.RBC.2020v66n1.474
}

\author{
Symptom Pattern in Colorectal Cancer Patients according to Age \\ Patrón de Síntomas en Pacientes con Cáncer Colorrectal según la Edad
}

Silmara Fernandes Moura'; Marianne Regina Silva Potengy de Mello²; Camila Drumond Muzi; Raphael Mendonça Guimarães ${ }^{4}$

Resumo

Introduçáo: $\mathrm{O}$ câncer colorretal está entre os tipos de câncer mais comuns na população brasileira e mundial com altos índices de mortalidade. Alguns estudos mostram que há uma diferença de carga sintomatológica para esse tipo de câncer entre adultos jovens e idosos que, consequentemente, podem deteriorar a qualidade de vida nesses pacientes. Objetivo: Investigar a diferença no padrão de sintomas entre pacientes adultos e idosos com câncer de cólon e reto. Método: Estudo transversal que utilizou um conjunto de dados sobre a prevalência de sintomas entre pacientes com câncer colorretal atendidos no Instituto Nacional de Câncer José Alencar Gomes da Silva (INCA), por meio de um inventário de sintomas recentemente adaptado para o Brasil. Resultados: Foram entrevistados 348 pacientes, destes, 101 pacientes eram adultos jovens $(29,1 \%)$ e 247 idosos $(70,9 \%)$. Obteve-se diferença entre os sintomas analisados para dor $(\mathrm{p}=0,033)$, nervosismo $(\mathrm{p}=0,013)$, sonolência $(\mathrm{p}=0,033)$, tristeza $(\mathrm{p}=0,003)$, problemas com desejo sexual ou atividade sexual $(\mathrm{p}=0,014)$, falta de apetite $(\mathrm{p}=0,028)$, irritaçáo $(\mathrm{p}=0,013)$, mudança nos gostos dos alimentos $(\mathrm{p}=0,042)$, perda de cabelo $(\mathrm{p}=0,002)$ e "eu não pareço mais o mesmo" ( $\mathrm{p}<0,001)$. Conclusáo: A carga sintomatológica frente ao câncer colorretal pode apresentar distinção conforme a idade. Isso é relevante, pois reforça a ideia de individualizar o tratamento para melhorar a assistência e, consequentemente, a qualidade de vida desses doentes.

Palavras-chave: Neoplasias Colorretais; Qualidade de Vida; Avaliação de Sintomas; Adulto; Idoso.

\begin{abstract}
Introduction: Colorectal cancer is among the most common types of cancer in the Brazilian and world population, with high rates of mortality. Some studies show that there is a difference in the symptomatic burden for this cancer among young adults and elderly individuals, which can deteriorate the quality of life of these patients. Objective: To investigate the difference in the pattern of symptoms among young adults and elderly patients with colon and rectum cancer. Method: Cross-sectional study using dataset on the prevalence of symptoms among colorectal cancer patients attended at the National Cancer Institute José Alencar Gomes da Silva (INCA) through a recently adapted inventory of symptoms for Brazil. Results: A total of 348 patients were interviewed, of which 101 were young adults $(29,1 \%)$ and $247(70.9 \%)$ were elderly individuals. There was a difference between the symptoms analyzed for pain $(p=0.033)$, nervousness $(p=0.013)$, drowsiness $(\mathrm{p}=0.033)$, sadness $(\mathrm{p}=0.003)$, problem with sexual interest or activity $(\mathrm{p}=0.014)$, loss of appetite $(\mathrm{p}=0.028)$, irritation $(\mathrm{p}=0.013)$, change of food taste ( $\mathrm{p}=0.042)$, hair loss ( $\mathrm{p}=0.002$.) and "I don't look like myself" $(\mathrm{p}<0.001)$. Conclusion: The symptomatic burden of colorectal cancer may differ according to age. This is relevant because reinforces the idea of individualizing the treatment to improve the care and, consequently, the quality of life of these patients.
\end{abstract}

Key words: Colorectal Neoplasms; Quality of Life; Symptoms Assessment; Adult; Aged.
Resumen

Introducción: El cáncer colorrectal se encuentra entre los tipos de cáncer más comunes em la población brasileńa y mundial, con altas tasas de mortalidad. Algunos estudios muestran que existe una diferencia en la carga sintomática para este tipo de cáncer entre adultos jóvenes y viejos, lo que en consecuencia puede deteriorar la calidad de vida en estos pacientes. Objetivo: Investigar la diferencia en el patrón de síntomas entre pacientes adultos y ancianos con cáncer de colon y recto. Método: Estudio transversal que utiliza un conjunto de datos de prevalencia de síntomas entre pacientes con cáncer colorrectal tratados en el Instituto Nacional del Cáncer José Alencar Gomes da Silva (INCA) a través de un inventario de síntomas recientemente adaptado para o Brasil. Resultados: Se entrevisto a 348 pacientes, de estos 101 pacientes eran adultos jóvenes (29,1\%) y 247 en acianos $(70,9 \%)$. Hubo una diferencia entre los síntomas analizados para el dolor $(\mathrm{p}=0,003)$, nerviosismo $(\mathrm{p}=0,013)$, somnolencia $(\mathrm{p}=0,033)$, tristeza $(\mathrm{p}=0,003)$, problemas con el deseo sexual o actividad sexual $(\mathrm{p}=0,014)$, falta de apetito $(\mathrm{p}=0,028)$, irritación $(\mathrm{p}=0,013)$, cambio en los gustos de los alimentos $(\mathrm{p}=0,042)$, pérdida de cabello $(\mathrm{p}=0,002)$ y "Ya no me veo igual” $(\mathrm{p}<0,001)$. Conclusión: La carga sintomática del cáncer colorrectal se puede distinguir según la edad. Esto es relevante porque refuerza la idea de individualizar el tratamiento para mejorar la atención y, en consecuencia, la calidad de vida de estos pacientes.

Palabras clave: Neoplasias Colorrectales; Calidad de Vida; Evaluación de Síntomas; Adulto; Anciano.

\footnotetext{
${ }^{1}$ Universidade Federal do Estado do Rio de Janeiro. Escola de Medicina e Cirurgia. Rio de Janeiro (RJ), Brasil. Orcid iD: https://orcid.org/0000-0002-2121-5321 ${ }^{2}$ Universidade Federal do Estado do Rio de Janeiro. Escola de Medicina e Cirurgia. Rio de Janeiro (RJ), Brasil. Orcid iD: https://orcid.org/0000-0001-9364-1656 ${ }^{3}$ Instituto Nacional de Câncer José Alencar Gomes da Silva. Rio de Janeiro (RJ), Brasil. Orcid iD: https://orcid.org/0000-0002-5567-0437

${ }^{4}$ Fundação Oswaldo Cruz. Escola Politécnica de Saúde Joaquim Venâncio. Rio de Janeiro (RJ), Brasil. Orcid iD: https://orcid.org/0000-0003-1225-6719

Endereço para correspondência: Raphael Mendonça Guimarães. Avenida Brasil, 4365 - Manguinhos. Rio de Janeiro (RJ), Brasil. CEP 21040-900. E-mail: raphael.guimaraes@fiocruz.br
} 


\section{INTRODUÇÃO}

O câncer colorretal está entre os tipos de câncer mais comuns na populaçáo brasileira e mundial. Dados da International Agency for Research on Cancer ${ }^{1}$ de 2018 estimaram que o câncer colorretal deverá aumentar cerca de $75 \%$, ultrapassando mais de 11,4 milhóes de novos casos e mais de 6,1 milhões de mortes até o ano de $2040^{1}$. O Instituto Nacional de Câncer José Alencar Gomes da Silva (INCA) ${ }^{2}$, para o biênio 2018-2019, estimou a incidência para o Brasil de 17.380 novos casos no sexo masculino e 18.980 no sexo feminino, sendo o terceiro tipo de câncer mais frequente entre os homens e o segundo entre mulheres no país ${ }^{2}$. Os sintomas relacionados dependerão da localização do câncer e da gravidade do caso. Gosselin et al. ${ }^{3}$ descreveram os sintomas e sua intensidade nos pacientes com câncer de cólon e reto, observando que aqueles mais prevalentes foram dispneia, náusea, inapetência, constipação, diarreia, dor, fadiga, sensação de cansaço e insônia. A gravidade de cada caso dependerá do tipo histológico do câncer e do seu estadiamento, influenciando na escolha do tipo de tratamento ${ }^{4}$.

Apesar dos avanços da medicina acerca do tratamento do câncer, os resultados entre os pacientes mais idosos são considerados menos promissores quando comparados com os pacientes mais jovens. Yates et al..$^{5}$ mostraram que os pacientes oncológicos mais velhos relataram um padrão sintomatológico diferenciado. Apesar disso, os pacientes mais velhos relataram menos severidade e frequência dos sintomas, e menos angústia em relação ao tratamento ${ }^{6}$. Essa diferença de resposta ao tratamento náo é totalmente compreendida na literatura, porém alguns estudos citam como sendo consequência da carga sintomática maior nos mais velhos, da menor adesão ao tratamento, além do impacto físico e mental em resposta ao tratamento do câncer serem maiores nesses pacientes. A fim de contribuir com essa análise, o objetivo do presente estudo foi estimar a diferença na ocorrência de sintomas em pacientes com câncer colorretal de acordo com a faixa etária.

\section{MÉTODO}

Trata-se de estudo transversal que utilizou um conjunto de dados sobre a prevalência de sintomas entre pacientes com câncer colorretal atendidos no INCA. A amostra, obtida por conveniência, incluiu 348 pacientes adultos internados no Serviço de Cirurgia Abdominopélvica, entre 2016 e 2018 , equivalente a $82 \%$ dos pacientes internados naquele período.

Os critérios de inclusão foram pacientes com idade igual ou superior a 18 anos, portadores de câncer colorretal, internados no Hospital do Câncer I (HCI)/
INCA, independente do estadiamento. Os critérios para exclusão foram pacientes que apresentavam distúrbios cognitivos, avaliados por meio do miniexame do estado mental (MEEM), ou em condiçóes capazes de comprometer a veracidade das respostas, com neoplasia ou metástase para sistema nervoso central (SNC), cujas informaçóes foram obtidas por dados de prontuário.

A coleta foi realizada com a aplicaçáo da escala Memorial Sypmtoms Assessment Scale (MSAS-BR) em forma de entrevista individual após prévia informação sobre os objetivos da pesquisa, concordância em participar do estudo e assinatura do Termo de Consentimento Livre e Esclarecido. O MSAS-BR é uma ferramenta de avaliação de 32 sintomas feito para medir a prevalência e as três características associadas a eles (frequência, gravidade e angústia), englobando aspectos físicos e psicológicos experimentados por pacientes oncológicos na semana anterior à entrevista. Esse instrumento foi validado para pacientes oncológicos no contexto brasileiro e avaliado quanto à sua consistência por estudos anteriores ${ }^{7,8}$. Para a coleta de dados adicionais, foi empregado um formulário elaborado pelos autores, incluindo dados sociodemográficos e clínicos como: idade, sexo, estado civil, nível de escolaridade, raça, diagnóstico primário, estadiamento, uso de dispositivos e local de tratamento (ambulatorial e internação). Essas informações foram obtidas por entrevista com os pacientes.

Para estimar a associaçáo entre a ocorrência dos sintomas e a idade, foram obtidos dois grupos: adultos, entre 18 e 59 anos; e idosos, com 60 anos ou mais. Para avaliar a diferença estatística para variáveis categóricas, foi utilizado o teste de qui-quadrado de Pearson e, para as variáveis contínuas, o teste de análise de variância Anova. Foi aceito um nível de significância estatística de $95 \%$. As análises de dados foram realizadas utilizando o Statistical Package for the Social Sciences (SPSS), versão 22.

Este estudo cumpriu as especificaçóes éticas e legais da Resolução n. ${ }^{0}$ 466/12, do Conselho Nacional de Saúde/ Ministério da Saúde, e possui autorização do Comitê de Ética em Pesquisa (CEP) do INCA, por meio do parecer consubstanciado número 863.339.

\section{RESULTADOS}

Foram entrevistados 348 pacientes, sendo 101 indivíduos adultos jovens e 247 idosos. A Tabela 1 apresenta a caracterizaçáo desses pacientes. Percebe-se que a amostra era composta, em sua maioria, por homens casados, brancos, com escolaridade de nível médio, tumores de localizaçáo colônica, moderadamente diferenciados, em estadiamento III. Não houve diferença dessas características entre os pacientes idosos e adultos. 
Tabela 1. Características clínicas e demográficas da amostra de estudo (n=348)

\begin{tabular}{|c|c|c|c|c|c|}
\hline \multirow{3}{*}{ Variáveis } & \multicolumn{4}{|c|}{ Idade } & \multirow{3}{*}{ p valor } \\
\hline & \multicolumn{2}{|c|}{$\begin{array}{c}\text { Adultos jovens }(<60 \\
\text { anos) }(n=101)\end{array}$} & \multicolumn{2}{|c|}{$\begin{array}{l}\text { Idosos } \\
\text { (60 anos e mais) }(n=247)\end{array}$} & \\
\hline & $\mathbf{n}$ & $\%$ & $\mathbf{n}$ & $\%$ & \\
\hline \multicolumn{6}{|l|}{ Sexo } \\
\hline Masculino & 56 & 54,99 & 137 & 55,51 & 0,98 \\
\hline Feminino & 45 & 45,01 & 110 & 44,49 & \\
\hline \multicolumn{6}{|l|}{ Estado Civil } \\
\hline Solteiro & 28 & 28,10 & 47 & 18,90 & \multirow{3}{*}{0,18} \\
\hline Casado & 61 & 60,30 & 165 & 66,60 & \\
\hline Viúvo & 12 & 11,40 & 36 & 14,50 & \\
\hline \multicolumn{6}{|l|}{ Raça } \\
\hline Branco & 62 & 60,90 & 157 & 63,40 & \multirow[t]{2}{*}{0,36} \\
\hline Negro/Pardo & 39 & 39,10 & 90 & 36,60 & \\
\hline \multicolumn{6}{|l|}{ Escolaridade } \\
\hline Fundamental & 35 & 35,00 & 96 & 39,00 & \multirow{3}{*}{0,29} \\
\hline Médio & 49 & 49,00 & 126 & 51,00 & \\
\hline Superior & 16 & 16,00 & 25 & 10,00 & \\
\hline \multicolumn{6}{|l|}{ Diferenciação tumoral } \\
\hline Bem diferenciado & 9 & 8,55 & 22 & 8,86 & \multirow{3}{*}{0,45} \\
\hline Moderadamente diferenciado & 57 & 56,42 & 156 & 63,13 & \\
\hline Mal diferenciado & 35 & 35,03 & 69 & 28,00 & \\
\hline \multicolumn{6}{|l|}{ Estadiamento } \\
\hline Estágios I/II & 48 & 47,66 & 109 & 44,08 & \multirow[t]{2}{*}{0,56} \\
\hline Estágios III/IV & 53 & 52,34 & 138 & 55,92 & \\
\hline \multicolumn{6}{|l|}{ Localização } \\
\hline Cólon & 58 & 57,43 & 156 & 63,30 & \multirow[t]{2}{*}{0,32} \\
\hline Reto & 43 & 42,57 & 91 & 36,70 & \\
\hline \multicolumn{6}{|l|}{ Metástase } \\
\hline Sim & 41 & 41,00 & 96 & 38,70 & \multirow[t]{2}{*}{0,38} \\
\hline Não & 60 & 59,00 & 151 & 61,30 & \\
\hline
\end{tabular}

A respeito dos sintomas, entre os 32 analisados pelo MSAS-BR, os sintomas mais prevalentes foram perda de peso, dor, boca seca e preocupaçóes. Os menos prevalentes foram feridas na boca, dificuldade para concentrar e para engolir (Tabela 2). De forma geral, para a maioria dos sintomas, não houve diferença na ocorrência entre os mais novos e os mais velhos. Entretanto, houve significância estatística para dor $(\mathrm{p}=0,033)$, nervosismo $(\mathrm{p}=0,013)$, sonolência $(\mathrm{p}=0,033)$, tristeza $(\mathrm{p}=0,003)$, problemas com desejo sexual ou atividade sexual $(\mathrm{p}=0,014)$, falta de apetite $(\mathrm{p}=0,028)$, irritação $(\mathrm{p}=0,013)$, mudança nos gostos dos alimentos ( $\mathrm{p}=0,042)$, perda de cabelo $(\mathrm{p}=0,002)$ e "eu náo pareço mais o mesmo" $(\mathrm{p}<0,001)$.

\section{DISCUSSÃO}

A carga sintomática frente à doença e ao tratamento do câncer difere segundo a faixa etária de ocorrência e, por muitas vezes, tal diferença é negligenciada diante dos cuidados, resultando em uma promoção débil de qualidade de vida a esses pacientes 9 . No que se refere à diferença em relação as faixas etárias estudadas, observa-se que, quando houve diferenças, há mais marcadamente queixas de cunho psíquico em pacientes idosos, quando comparados aos mais jovens. Parte disso é explicado pelo fato de os idosos trazerem consigo uma carga maior de doenças prévias, o que gera medo da morte 
Tabela 2. Descrição dos sintomas em pacientes com câncer colorretal de acordo com a faixa etária (n=348)

\begin{tabular}{|c|c|c|c|c|c|c|c|c|c|}
\hline \multirow{3}{*}{ Item } & \multicolumn{4}{|c|}{ Adultos jovens } & \multicolumn{4}{|c|}{ Idosos } & \multirow{3}{*}{ p valor } \\
\hline & \multicolumn{2}{|c|}{ Sim } & \multicolumn{2}{|c|}{ Não } & \multicolumn{2}{|c|}{ Sim } & \multicolumn{2}{|c|}{ Não } & \\
\hline & $\mathbf{n}$ & $\%$ & $\mathbf{n}$ & $\%$ & $\mathbf{n}$ & $\%$ & $\mathbf{n}$ & $\%$ & \\
\hline Dificuldade para se concentrar & 6 & 5,94 & 95 & 94,06 & 15 & 6,07 & 232 & 93,93 & 0,663 \\
\hline Dor & 61 & 60,40 & 40 & 39,60 & 136 & 55,06 & 111 & 44,94 & 0,033 \\
\hline Falta de energia & 48 & 47,52 & 53 & 52,48 & 117 & 47,37 & 130 & 52,63 & 0,979 \\
\hline Tosse & 12 & 11,88 & 89 & 88,12 & 28 & 11,34 & 219 & 88,66 & 0,885 \\
\hline Nervosismo & 32 & 31,68 & 69 & 68,32 & 73 & 29,55 & 174 & 70,45 & 0,013 \\
\hline Boca seca & 54 & 53,47 & 47 & 46,53 & 132 & 53,44 & 115 & 46,56 & 0,997 \\
\hline Enjoo & 45 & 44,55 & 56 & 55,45 & 110 & 44,53 & 137 & 55,47 & 0,997 \\
\hline Sonolência & 38 & 37,62 & 63 & 62,38 & 97 & 39,27 & 150 & 60,73 & 0,033 \\
\hline $\begin{array}{l}\text { Dormência ou formigamento nas mãos/ } \\
\text { pés }\end{array}$ & 21 & 20,79 & 80 & 79,21 & 51 & 20,65 & 196 & 79,35 & 0,976 \\
\hline Dificuldade para dormir & 40 & 39,60 & 61 & 60,40 & 99 & 40,08 & 148 & 59,92 & 0,934 \\
\hline Empanzinamento & 48 & 47,52 & 53 & 52,48 & 118 & 47,77 & 129 & 52,23 & 0,966 \\
\hline Problemas para urinar & 11 & 10,89 & 90 & 89,11 & 27 & 10,93 & 220 & 89,07 & 0,991 \\
\hline Vômitos & 24 & 23,76 & 77 & 76,24 & 58 & 23,48 & 189 & 76,52 & 0,955 \\
\hline Falta de ar & 14 & 13,86 & 87 & 86,14 & 34 & 13,77 & 213 & 86,23 & 0,981 \\
\hline Diarreia & 13 & 12,87 & 88 & 87,13 & 31 & 12,55 & 216 & 87,45 & 0,935 \\
\hline Tristeza & 43 & 42,57 & 58 & 57,43 & 112 & 45,34 & 135 & 54,66 & 0,003 \\
\hline Suor & 12 & 11,88 & 89 & 88,12 & 29 & 11,74 & 218 & 88,26 & 0,971 \\
\hline Preocupações & 53 & 52,48 & 48 & 47,52 & 129 & 52,23 & 118 & 47,77 & 0,966 \\
\hline $\begin{array}{l}\text { Problemas com o desejo ou atividade } \\
\text { sexual }\end{array}$ & 22 & 21,78 & 79 & 78,22 & 49 & 19,84 & 198 & 80,16 & 0,014 \\
\hline Coceira & 13 & 12,87 & 88 & 87,13 & 32 & 12,96 & 215 & 87,04 & 0,983 \\
\hline Falta de apetite & 43 & 42,57 & 58 & 57,43 & 113 & 45,75 & 134 & 54,25 & 0,028 \\
\hline Tontura & 20 & 19,80 & 81 & 80,20 & 50 & 20,24 & 197 & 79,76 & 0,926 \\
\hline Dificuldade para engolir & 10 & 9,90 & 91 & 90,10 & 24 & 9,72 & 223 & 90,28 & 0,958 \\
\hline Irritação & 29 & 28,71 & 72 & 71,29 & 76 & 30,77 & 171 & 69,23 & 0,013 \\
\hline Feridas na boca & 2 & 1,98 & 99 & 98,02 & 5 & 2,02 & 242 & 97,98 & 0,979 \\
\hline Mudança no gosto dos alimentos & 22 & 21,78 & 79 & 78,22 & 58 & 23,48 & 189 & 76,52 & 0,042 \\
\hline Perda de peso & 68 & 67,33 & 33 & 32,67 & 167 & 67,61 & 80 & 32,39 & 0,959 \\
\hline Perda de cabelo & 17 & 16,83 & 84 & 83,17 & 37 & 14,98 & 210 & 85,02 & 0,002 \\
\hline Prisão de ventre & 30 & 29,70 & 71 & 70,30 & 73 & 29,55 & 174 & 70,45 & 0,978 \\
\hline Inchaço nos braços ou pernas & 26 & 25,74 & 75 & 74,26 & 63 & 25,51 & 184 & 74,49 & 0,963 \\
\hline "Eu não pareço mais eu mesmo(a)" & 50 & 49,50 & 51 & 50,50 & 114 & 46,15 & 133 & 53,85 & $<0,001$ \\
\hline Alterações na pele & 32 & 31,68 & 69 & 68,32 & 79 & 31,98 & 168 & 68,02 & 0,956 \\
\hline
\end{tabular}

por pensamentos de fim de vida. A esse respeito, Rao et al. ${ }^{10}$, ao compararem pacientes com câncer e pacientes com doenças crônicas sem câncer em relação a um grupo controle de pessoas saudáveis, demostraram que pacientes com câncer e pacientes com doenças crônicas tinham um risco aumentado para apresentar sintomas psicológicos e que, quando somados - câncer e doenças crônicas -, aumentavam ainda mais o risco para desenvolvimento de tal perfil. Thong et al. ${ }^{9}$ reforçam essa tese, afirmando que pacientes com multicomorbidades tendem a ter mais queixas psicológicas em relação àqueles sem comorbidades.

Entre pacientes mais jovens, nota-se que as queixas físicas foram mais frequentes naqueles com diferença significativa quando comparados aos idosos. Sobre a dor, 
os jovens tenderam a ser mais queixosos. Isso se deve, possivelmente, ao limite de percepção dolorosa, pois, em razão da carga de comorbidades prévias associadas e, por consequência, de maiores experiências prévias em relação à dor, que, de modo geral, se relacionam mais com os idosos, fazem com que eles sejam mais resilientes no que diz respeito à autopercepçáo da dor do que os jovens ${ }^{9}$. Percebe-se, também, que a alteração da autoimagem e do estilo de vida durante o processo da doença pode ter sido um fator contribuinte para queixas como nervosismo, problemas com desejo ou atividade sexual, perda de cabelo e "eu não pareço mais o mesmo" serem mais significativas nesse grupo, uma vez que mudanças sociais e estilo de vida têm um impacto maior nos jovens. Esse resultado é corroborado por Barr e Feeny ${ }^{11}$, que acrescentam à discussão a forma de o tratamento do câncer colorretal envolver o uso de bolsa de colostomia, o que costuma afetar negativamente a autoimagem do paciente por ser considerado um "constrangimento social"11.

De forma geral, nota-se que a experiência de um paciente frente ao câncer gera múltiplos sintomas coocorrentes tanto psicológicos quanto orgânicos, que influenciam de forma negativa na qualidade de vida. Fatores psicossociais, como a idade, podem interferir nas queixas apresentadas e na carga sintomática diante da doença ${ }^{12}$. No que concerne sobre a qualidade de vida nesses pacientes, estudos ainda destacam que, em virtude desse conceito abrangente e complexo, há divergências sobre qual faixa etária tem a qualidade de vida mais comprometida frente ao câncer colorretal. Dessa forma, o reconhecimento de sintomas específicos e a sua frequência entre as faixas etárias são de extrema importância.

\section{CONCLUSÃO}

Observa-se que a carga sintomatológica frente ao câncer colorretal pode apresentar distinção conforme a idade, havendo sintomas psicológicos maior nos idosos do que entre os adultos jovens, e impactos sociais adversos e sintomas físicos influenciaram mais em indivíduos mais jovens. Dessa forma, individualizar o tratamento por meio do reconhecimento sintomatológico e ponderar quais são mais susceptíveis de acordo com a idade são estratégias relevantes para a gestão do cuidado.

\section{CONTRIBUIÇÕES}

Silmara Fernandes Moura e Marianne Regina Silva Potengy de Mello participaram da coleta de dados, análise e redação do manuscrito final. Camila Drumond Muzi e Raphael Mendonça Guimarães participaram da concepção do estudo, análise dos dados, redação e revisão final do manuscrito. Todos os autores aprovaram a versão final a ser publicada.

\section{DECLARAÇÃO DE CONFLITOS DE INTERESSES}

Não há.

\section{FONTES DE FINANCIAMENTO}

Bolsas de Iniciação Científica do Conselho Nacional de Desenvolvimento Científico e Tecnológico (CNPq)

\section{REFERÊNCIAS}

1. Bray F, Ferlay J, Soerjomataram I, et al. Global cancer statistics 2018: GLOBOCAN estimates of incidence and mortality worldwide for 36 cancers in 185 countries. CA Cancer J Clin. 2018;68(6):394-424. doi: https://doi. org/10.3322/caac. 21492

2. Instituto Nacional de Câncer José Alencar Gomes da Silva. Estimativa 2018: incidência de câncer no Brasil [Internet]. Rio de Janeiro: INCA; 2017. [acesso 2018 dez. 29]. Disponível em: http://www1.inca.gov.br/ estimativa/2018/

3. Gosselin TK, Beck S, Abbott DH, et al. The symptom experience in rectal cancer survivors. J Pain Symptom Manage. 2016;52(5):709-18. doi: https://doi. org/10.1016/j.jpainsymman.2016.05.027

4. Jeong G, Kim K, Kwak Y. Quality of life in colorectal cancer patients according to the severity of symptom clusters classification. Asian Oncol Nurs. 2014;14(2):7483. doi: https://doi.org/10.5388/aon.2014.14.2.74

5. Yates P, Miaskowski C, Cataldo JK, et al. Differences in composition of symptom clusters between older and younger oncology patients. J Pain Symptom Manage. 2015;49(6):1025-34. doi: https://doi.org/10.1016/j. jpainsymman.2014.11.296

6. Boeira SF, Guimarães RM, Acioli LR, et al. Cluster de sintomas e câncer na pesquisa em enfermagem: revisão sistemática. Rev Bras Cancerol. 2014;60(4):35161. doi: https://doi.org/10.32635/2176-9745. RBC.2014v60n4.462

7. Rocha LF, Carvalho MS, Lacerda AAM, et al. Equivalência semântica da versão em português do instrumento Memorial Symptom Assessment Scale (MSAS) para avaliar sintomas em pacientes oncológicos. Rev Bras Pesq Saúde.2017;19(2):83-91. doi: https://doi.org/10.21722/ rbps.v19i2.20584

8. Menezes JR, Luvisaro BMO, Rodrigues CF, et al. Confiabilidade teste-reteste da versão Brasileira do instrumento Memorial Symptom Assessment Scale para avaliação de sintomas em pacientes oncológicos. Einstein (São Paulo). 2017;15(2):148-54. doi: https:// doi.org/10.1590/s1679-45082017ao3645 
9. Thong MSY, Koch-Gallenkamp L, Jansen L, et al. Age-specific health-related quality of life in long-term and very long-term colorectal cancer survivors versus population controls: a population-based study. Acta Oncol. 2019;58(5):801-10. doi: https://doi.org/10.10 80/0284186X.2018.1557340

10. Rao WW, Yang MJ, Cao BN, et al. Psychological distress in cancer patients in a large Chinese cross-sectional study. J Affect Disord. 201915;245:950-6. doi: https://doi. org/10.1016/j.jad.2018.11.089

11. Barr RD, Feeny DA. Health-related quality of life in adolescents and young adults with cancer - including a focus on economic evaluation. Pediatr Blood Cancer. 2019;66(8):e27808. doi: https://doi.org/10.1002/ pbc. 27808

12. Decoster L, Quinten C, Kenis C, et al. Health related quality of life in older patients with solid tumors and prognostic factors for decline. J Geriatr Oncol. 201910(6):895-903. doi: https://doi.org/10.1016/j. jgo.2019.03.018 